\title{
Surface Rotation Correction and Strain Precision of Wide-Angle 2D DIC for Field Use
}

\author{
Halding, Philip Skov; Christensen, Christian O.; Schmidt, Jacob W.
}

Published in:

Journal of Bridge Engineering

Link to article, DOI:

10.1061/(ASCE)BE.1943-5592.0001358

Publication date:

2019

Document Version

Peer reviewed version

Link back to DTU Orbit

Citation (APA):

Halding, P. S., Christensen, C. O., \& Schmidt, J. W. (2019). Surface Rotation Correction and Strain Precision of Wide-Angle 2D DIC for Field Use. Journal of Bridge Engineering, 24(4), [04019008].

https://doi.org/10.1061/(ASCE)BE.1943-5592.0001358

\section{General rights}

Copyright and moral rights for the publications made accessible in the public portal are retained by the authors and/or other copyright owners and it is a condition of accessing publications that users recognise and abide by the legal requirements associated with these rights.

- Users may download and print one copy of any publication from the public portal for the purpose of private study or research.

- You may not further distribute the material or use it for any profit-making activity or commercial gain

- You may freely distribute the URL identifying the publication in the public portal 


\title{
SURFACE ROTATION CORRECTION AND STRAIN PRECISION OF WIDE-ANGLE
}

\section{D DIC FOR FIELD USE}

\author{
Philip S. Halding ${ }^{1}$, Christian O. Christensen ${ }^{2}$, and Jacob W. Schmidt ${ }^{3}$ \\ ${ }^{1}$ Assistant professor, Technical University of Denmark, Brovej 118, 2800 Kgs. Lyngby, Denmark. \\ Email:phsh@byg.dtu.dk \\ ${ }^{2} \mathrm{PhD}$-student, Technical University of Denmark, Brovej 118, 2800 Kgs. Lyngby, Denmark. \\ ${ }^{3}$ Associate professor, Technical University of Denmark, Brovej 118, 2800 Kgs. Lyngby, Denmark.
}

\section{ABSTRACT}

The paper describes how 2D Digital Image Correlation is used on underneath surfaces of concrete bridges with wide-angle lens camera during load testing, and how it has potential as a stop criterion in proof loadings.

A method is proposed for correction of out-of-plane deflection including rotation of the surface. The method is applied to laboratory tests, using well defined circular speckle patterns, as well as to a field tested bridge (on raw concrete). The proposed correction corresponds to the level of pseudo strain, but is very sensitive to precise surface deflection measurements.

In the laboratory tests, a strain precision of the wide-angle lens camera is compared to a regular lens camera. The parametric study concludes that a Pattern Pixel Relation, in the interval from 4 to 9 pixels per pattern circle diameter, provides the optimal precision regardless of the camera type.

The field tested bridge has less good precision compared to most parameter combinations of the laboratory tests. Nevertheless, the field strain precision has potential for improvement based on learnings from the laboratory tests. 


\section{INTRODUCTION}

25 The following main approaches are typically used to monitor surface strains on concrete structures: i)

26 Direct contact measurements, which are used to measure in direct contact with the surface (by e.g. strain

27 gauges, extensometers, etc.) or ii) non-contact measurements (by e.g. photogrammetry, laser scanning,

Digital Image Correlation etc.) placed in a distance from the structure. Such equipment can potentially be used for structural health monitoring, diagnostic loading, local strain evaluations, laboratory testing etc.

Proof load testing of existing concrete bridges is an extensive monitoring challenge, due to the environmental exposure of the monitoring equipment, and limited testing time. Such application can require simultaneous monitoring of a large number of locations, and that different monitoring sources are used to ensure a robust identification of unique stop criteria with sufficient precision. The precision of the output should be of a quality, which can be used to update theoretical models related to the capacity- or probabilistic evaluations. Furthermore, fast mounting and dismantling of the monitoring equipment is necessary to reduce traffic disturbance as much as possible.

This paper focuses mainly on the use of wide-angle Digital Image Correlation (DIC), which is a noncontact methods that seems promising and useful as a mean to solve some of these challenges. Such equipment can be fast to apply, and can potentially provide full field strain measurements of larger structural surfaces. The use of wide-angle cameras have, to the author's knowledge, not been used for DIC evaluations before, but is deemed to be an essential tool for evaluation of strains and cracks on large concrete surfaces.

DIC is an advanced method for evaluation of deformations on a test specimen by the use of digital measurements with one camera) and 3D evaluations (out-of-plane measurements with two or more cameras), where 3D DIC always, to the knowledge of the authors, require a more time consuming and 
In the literature, 2D DIC is predominantly used for relatively small plane surfaces, where no out-of-

51 plane deflection is expected to occur during testing, and where the surface is parallel to the direction of the 52 image sensor of the camera (e.g. Wang et al. (2011), Hoult et al. (2013), Pan et al. (2014), and Chen et al. 53 (2015)).

Although some researchers have addressed the correction of out-of-plane deflection of 2D DIC (Schreier et al. (2009)), no one has studied methods for correction of out-of-plane deflection on large in-situ concrete surfaces, where surface rotations are present as well, and where application of painted speckles can be difficult to apply due to the site conditions, surface accessibility, size of the surface, and available time.

This paper proposes a strain evaluation method, which takes surface rotations into account, when correcting for out-of-plane deflection on large surfaces. The method was tested under laboratory conditions and applied to a real load tested concrete bridge in Denmark, as an example. The bridge was a short span

61 (less than $12 \mathrm{~m}$ ), simply supported, highway beam bridge, where DIC-cameras were applied to the underneath bridge deck surface. Evaluation of strains, based on conventional out-of-plane displacement correction methods alone, seems inapplicable in the case, since it differs significantly due to the additional surface rotation. The discrepancies between the scenarios is depicted in Figure 1.

Furthermore, the paper includes a comparing evaluation of the precision applied to both large scale laboratory tests, and field tests, where a wide-angle lens DSLR-camera was used, as a state-of-the-art DIC equipment, to achieve the largest possible surface for evaluation.

\section{D Digital Image Correlation - precision and errors}

The principle of DIC is that captured digital photographs are evaluated by dividing them into subsets of

71 a certain size (e.g. 80x80 pixels). These subsets are assessed in terms of the grey level. A DIC-software can

72 distinguish the subsets from each other, and they are tracked continuously during the surface deformation

73 to determine the direction and size of strains and displacements. Interpolation done by the DIC-software

74 provides precision at sub pixel level (Bruck et al. (1989)) and different researchers have aimed to optimize 
the correlation computation method to achieve precisions as good as 1/10 to 1/20 of a pixel (Ruocci et al. (2016)).

The precision of DIC can be affected by numerous influencing parameters (Lecompte et al. (2006 and 2007), Bornert et al. (2009), Triconnet et al. (2009)), such as:

a) The surface texture (must have a distinct high contrast pattern). The standard method in the literature is to manually apply painted speckles, even though few researchers have applied DIC without a painted pattern (Waterfall et al. (2014) and Schmidt et al. (2014)). Painted speckles on concrete bridges for in-situ tests have also been applied in rare occations (Yoneyama and Kitagawa (2007) and Halding et al. (2016)), but the creation of painted speckles on larger surfaces is deemed very time consuming (Sutton et al. (2017)). The speckles on a concrete bridges by Halding et al. (2016) were monitored at close proximity to the bridge surface, and in the investigation by Yoneyama and Kitagawa (2007), it was from a far distance.

b) The subset size. The choice of subset size has been discussed throughout the literature for small scale laboratory tests (e.g. Crammond et al. (2013), and Park et al. (2017)). A general conclusion is that larger subsets generate a better precision when evaluating the strain. However, when evaluating crack initiations smaller subsets should be used.

c) The subset size to speckle size relation. It is deemed that the precision is highly dependent on this relation but it is still an open question, how these mutually affect each other for in-situ tests on raw concrete surfaces. In addition, the speckle sizes often vary on a painted surface, and similarly the texture roughness differ on raw concrete surfaces, which potentially means that the precision can vary depending on the identified subset. This seems not fully understood and may be essential when evaluating large surfaces.

For small scale experiments, Triconnet et al. (2009) proposed that the standard deviation of the grey level distribution within each subset should have a minimum value of 6 grey levels, and that the maximum speckle size should be 1/4 of the subset size. Later Sutton et al. (2017) stated that the number of pixels per speckle should not be less than three, to achieve adequate DIC interpolation. 
102 Pseudo strain errors from lens distortion and out-of-plane deflections (optical strain errors)

103 One recurring error in the literature is lens distortion. To correct the distorted image plane, different 104 methods have been suggested (e.g. Yoneyama et al. (2006)). Today, the commercial software Adobe 105 Photoshop (Photoshop 2018) have incorporated anti-lens-distortion algorithms for a large range of cameras 106 and lenses, and the authors have previously checked the correctness of lens distortion correction via this 107 method (Halding et al. (2018)).

108 In regard to out-of-plane deflections (pure translation towards the camera), Hoult et al. (2013) did an 109 experimental study on thin steel specimens in tension, with cameras for 2D DIC on both sides of the 110 specimens. They showed, by incremental movement of the cameras, that errors from out-of-plane 111 deflections had major influence on the measured strain, and summed up a number of solutions from their 112 own research and from the literature: i) By calculated correction based on geometrical consideration (from 113 Schreier et al. (2009)), ii) by minimization of the problem by increasing the distance to the camera, iii) by 114 estimating the error from knowledge of the Poisson's ratio of the material, iv) by using cameras on both 115 sides of the specimen, v) and by comparing to an object without deflection next to the specimen.

116 Only i) is applicable for in-situ load tests of concrete bridges by DIC of the underneath surface. The 117 authors have also previously investigated a calculation similar to i) that is solely valid, where no surface 118 rotation occur (Halding et al. (2018)), see Figure 2:

$$
L_{d}=\frac{L \cdot h}{h-n}
$$

$119 L_{d}$ is the detected distance between two points on the evaluated surface after out-of-plane deflection has 120 occurred, and $L$ is the original distance between the points before deflection. The out-of-plane deflection is 121 denoted, $n$, while, $h$, is the camera to surface distance before deflection. The strain alteration from out-of122 plane deflection was then determined as:

$$
\varepsilon_{\text {oop }}=\frac{L_{d}-L}{L}=\frac{h}{h-n}-1
$$


The above is based on photographs without lens distortion, and the correction method applies in practice

124 in areas, where the surface rotation is insignificantly small during testing (for instance at the mid-span of a 125 simply supported, uniformly loaded beam).

126 For evaluation of larger surfaces by e.g. use of wide-angle lens DSLR-camera, the correction for out127 of-plane deflection is only part of the required total strain correction, when using 2D DIC. The inclination 128 (or rotation) of the deflected surface leads to an error as well.

\section{The full-scale concrete bridge field testing program}

The paper is part of a Danish bridge testing project that was initiated in 2016 by the project partners: The Danish Road Directorate, the Technical University of Denmark, and the consultancy firm COWI A/S.

133 The aim of the project is to develop a method for proof load testing of existing bridges with the purpose of 134 determining if the tested bridges can achieve a higher loading class than predicted by the use of established 135 theoretical methods. The developed method includes advanced monitoring of the bridge response (for stop 136 criteria), where 2D DIC is chosen as one of the most promising approaches to evaluate thresholds during 137 testing.

\section{CORRECTION FOR SURFACE ROTATION DURING OUT-OF-PLANE DEFLECTION}

140 In-situ tests of larger surfaces are in many cases difficult to perform without some level of out-of-plane 141 deflection- and rotation of the loaded specimen. For field applications, like bridge load testing with 142 evaluation of the underneath bridge surface, it is therefore required to extend the existing out-of-plane 143 correction calculation method to include the rotation of the deflected surface. This is, if it is not an option 144 to position the camera far away or to have a camera on both sides. For the following method to be applicable, 145 the out-of-plane deflection must be measured as well in several locations and with high accuracy. The total 146 correction method is employed in three tempi:

147 1. Correction for out-of-plane translation of the surface, based on measurements of the deflection. 
2. Correction for rotation of the surface, based on measurements of the deflection.

In Figure 3, the parameters for calculation of the surface rotation correction is presented.

The rotation of the surface is not corrected by the lens distortion correction since the viewed angle between two points on the surface will change from $\alpha$ to $\beta$ when the surface rotates. The perpendicular distance from camera to surface before deflection is denoted, $h$, and the deflection in point $\mathrm{A}$ is, $n$. The parameter, $x$, is the horizontal distance to the first point, B, on the surface, and, $L$, is the distance between $\mathrm{A}$ and $\mathrm{B}$. The change in deflection from B1 to B2 as the surface rotates around A is called $d n$. $d L$ is the horizontal change in position of point B. By trigonometry, $d L$, and the angles, $\alpha$ and $\beta$, are found as:

$$
\begin{gathered}
d L=L-\sqrt{L^{2}-d n^{2}} \\
\alpha=\arctan \left(\frac{L+x}{h-n}\right)-\arctan \left(\frac{x}{h-n}\right) \\
\beta=\arctan \left(\frac{L+x}{h-n}\right)-\arctan \left(\frac{x+d L}{h-n-d n}\right)
\end{gathered}
$$

The angles, $\alpha$ and $\beta$, can be utilized when determining the strain correction from the rotation on the lens surface, is the same as the relative change in distance between the points:

$$
\varepsilon_{\text {rot }}=\frac{\alpha-\beta}{\alpha}
$$

It should be noted that the above correction is for longitudinal strains in sections in straight line with the camera, which is what is investigated in this work. An extended version of the correction, for a 2D representation, could be developed by considering the component of the strain in the directions that are not 167 in line with the camera. 
The strain correction contributions ( $\varepsilon_{\text {oop }}$ and $\varepsilon_{\text {rot }}$ from Eq. (2) and Eq. (6)) must then be added to achieve the total correction. For a simply supported beam or deck, the largest strain error (pseudo compression)

171 caused by the rotation of the surface is found near the supports (position of maximum surface rotation), and

172 the maximum strain error (pseudo tension) from the deflection is found at mid-span (position of maximum 173 out-of-plane deflection). Hence, both correction contributions are essential in regard to analysis of full-field 174 studies of large areas. Figure 4 shows an example of such correction contributions.

175 To determine the true surface strain, the corrections must be subtracted from the directly measured DIC176 strains (from digital photographs without lens distortion):

$$
\varepsilon_{\text {true }}=\varepsilon_{D I C}-\varepsilon_{\text {rot }}-\varepsilon_{\text {oop }}
$$

\section{LABORATORY TESTS}

To compare the laboratory tests with an example from field load tests, the same DIC camera equipment was used in both cases. The overall purpose of the laboratory tests was to, in a controlled environment, provide a more standardized reference for the field tests as well as a direct comparison between a DSLRcamera with and without wide-angle lens. The standard of reference was regarding the strain precision, and the precision of the correction method. It should be noted that the strain analysis differs from crack initiation identification, where the optimal subset size is different.

\section{Test setup}

A Canon 6D with 20 Mpx (Megapixel) resolution and a wide-angle lens (Canon EF 16-35mm f/2.8L II USM), and a Canon 750D with 24 Mpx resolution and a regular lens (Canon EFS 18-55mm IS STM) was used in the tests. During the tests, the camera was positioned on a tripod facing a rigid steel frame with an 
192 pushed sideways in between horizontally positioned H-beams in the bottom and top of the frame, and there 193 was a small gap of approximately $10 \mathrm{~mm}$ between the top of the board and the flange of the top H-beam 194 after installation. The orientation of the camera was perpendicular to the non-deflected board surface in all 195 tests. The large board consisted of four smaller boards, connected by five horizontal laths, which were 196 screwed into the backside. The boards were made by 10 mm MDF (Mittel-Dichte Faserplatte: glued wood). 197 Foil strain gauges (HBM type: 10/120 LY11) and extensometers (Instron clip-on dynamic 2620-604) 198 for direct contact surface strain measurements were positioned on the front surface of the board as known 199 (discrete) references to the DIC strain measurements. The vertical distance between all gauges was 450 $200 \mathrm{~mm}$, and the strain gauges were glued to the surface after the layers of paint had carefully been grinded off 201 (a painted pattern had been applied in the laboratory tests). The extensometers were positioned next to the 202 strain gauges (100 mm horizontal gap) in the same height, and were being secured to the surface with elastic 203 bands through small holes drilled in the plate. Similarly, the wires from the gauges passed through drilled 204 holes in the plates to avoid too much interference with the DIC system.

205 See Figure 6 for the exact positions of the monitoring equipment. In Figure 7, photographs show the 206 setup from the front (top photograph), from the front zoomed in to two strain gauges and an extensometer 207 (bottom left photograph), and from the backside, where LVDT’s (Novotechnik 0-5 V) and bolts for manual 208 application of the deflection is seen (bottom right photograph).

209 The frame was designed so that deformations could be applied via bolts and nuts at mid-span on the 210 backside of the plates (via the columns) and generate a desired deflection of the plate. The boundary 211 conditions were assumed simply supported. LVDT's and dial gauges were positioned on the rear surface 212 as well, to measure the size of the deflection in a number of locations. The LVDT's measured in the same 213 location as the vertical strain gauges (on the front surface), while the dial gauges were positioned next to 214 where the bolts and nuts for deformation was applied. 


\section{Test specimens and grey levels}

217 Three MDF boards were painted to get background color \#949494 - grey nuance number 148 (number

218255 corresponds to pure white). A pattern of circle dots was then applied by spray paint - color \#585858

219 and grey nuance number 88 (number 0 corresponds to pure black) through perforated steel boards with

220 different sizes of holes for each board, see Figure 8. The perforated steel plates were positioned above the

221 boards, and the paint was sprayed through the holes:

222

Plate 1) Hole diameter $3 \mathrm{~mm}$, a hole-percentage of 33, and triangular hole distribution with center distance of $5 \mathrm{~mm}$

Plate 2) Hole diameter $5 \mathrm{~mm}$, a hole-percentage of 35, and triangular hole distribution with center distance of $8 \mathrm{~mm}$

Plate 3) Hole diameter $10 \mathrm{~mm}$, a hole-percentage of 40, and triangular hole distribution with center distance of $15 \mathrm{~mm}$

The hole-percentage is the area percentage of voids of the total plate area. The similar hole-percentages ensured that the area of each of the two grey nuances, and therefore the grey levels, ought to be comparable. chosen based on evaluation of concrete surfaces from digital photographs underneath two actual bridges during in-situ load testing in Denmark (one on a summer day, and one on a winter day). One representative photograph from both in-situ tests were utilized in determining grey levels for the boards in the laboratory tests. An average grey level histogram (based on all pixels of the whole photograph) for both in-situ

235 photographs was determined, and the nuances corresponding to the grey intensity at the 25 and 75 percentile

236 values of the histograms, see Figure 9, were chosen as the two nuances of the paint in the laboratory tests.

237 The type of histogram shown in the figure can be found via Photoshop, MatLab or similar programs. 


\section{Test procedure and test parameters}

Before each test, the level of light was measured in front of the camera lens (by handheld luxmeter: Extech HD400. With a precision of \pm 5 Lux). Each plate was then initially tested in non-deflected condition to achieve a strain-precision of the DIC-monitoring by capturing five Raw-format photographs of which two were chosen for evaluation in a DIC software. In theory, the measured surface strain should be zero from the first to the second photograph, but in practice, some erroneous strains occurred, which could be used in determining the precision under the chosen settings. The precision was calculated as the standard deviation of the strains in every point along a chosen section.

The board was then tested with an applied deflection of $10 \mathrm{~mm}, 20 \mathrm{~mm}$, and $30 \mathrm{~mm}$ at mid-span. The primary objective with deflection of the plate, was to be able to compare strain measurements from the DIC-system with strain gauge and extensometer measurements before and after performing the lens distortion correction, and the 2D out-of-plane deflection and surface rotation correction of the DIC-values.

The test parameters were: The camera distance, the subset size, the pattern circle diameter, and the camera type. The distance from camera to surface was set to $1.0 \mathrm{~m}, 2.6 \mathrm{~m}$, and $3.8 \mathrm{~m}$. The $2.6 \mathrm{~m}$ and $3.8 \mathrm{~m}$ distances were chosen since they were used in the in-situ tests as well.

In the post testing analysis, subset sizes of 40x40 pixels, 80x80 pixels and 120x120 pixels were investigated. All evaluations were performed with a point distance of $50 \%$ of the subset side length in the 256 calculations, and by using the software GOM Correlate (2018).

257 Information of the pattern circle diameters (3 $\mathrm{mm}, 5 \mathrm{~mm}$ and $10 \mathrm{~mm}$ ) and the camera types (normal- and 258 wide-angle lens) was presented earlier in the chapter, and the total test matrix is provided in Table 1 . The 259 matrix is similar for both camera types.

\section{DIC-PRECISION OF LABORATORY TESTS}

The readings of the level of light in front of the camera, before photographs were captured, showed to 
to board distance, the level of light was approximately $30 \%$ lower than the other distances, which did not result in a tendency of increase in the camera exposure time.

\section{Precision with no deflection (zero strain)}

The precision was determined, after correction of lens distortion, for all combinations of parameters, before deflection was applied to the tested surface. A vertical section was applied in the middle Region Of Interest (ROI), and the strain was determined in all measured points along the section. The standard deviation of these strains was then used as a precision quantity.

\section{The influence of the subset size and camera distance}

For strain precision evaluation, the precision was expected to improve as the subset size increased. Note that large subsets can be less appropriate in regard to crack detection (not within the scope of this work). Figure 10 shows the relationship between subset size and precision, and as expected, the precision improves with increasing subset size

The subset sizes were 40x40 pixels, 80x80 pixels, and 120x120 pixels, and the values at these subset sizes are joined with lines in the figure. This is to give an overview, and does not mean that there is a linear relationship between the measured points. The legend in the figure shows the different combinations of camera to surface distances (1.0 m, $2.6 \mathrm{~m}$ and $3.8 \mathrm{~m})$, and the circle pattern size ( $3 \mathrm{~mm}, 5 \mathrm{~mm}$ and $10 \mathrm{~mm}$ ). expected. Nevertheless, the camera distance is deemed to influence the PPR (Pattern Pixel Relation number of pixels per pattern circle diameter). The PPR is affected by both the camera to surface distance, the pattern size, as well as the specific camera specifications (e.g. the resolution). 
Figure 11 shows how the precision is influenced by the PPR of the detected surface. An example view of the pattern of PPR for different variations of parameters of the wide-angle lens camera is seen in Figure 12. Each photograph is zoomed to fit $50 \times 50$ pixels.

For all subset sizes, and for both cameras there is a significantly better precision for PPR in the interval

292

293 from 4 to 9 pixels per circle diameter, even though the surfaces were well detected by the camera and software. When the PPR increases beyond 9 (equivalent to a digital camera capturing photographs relatively close to the surface or a high camera resolution) or decreases below 4 (equivalent to a digital camera capturing photographs relatively far from the surface or a low camera resolution), the standard deviation increases.

In Figure 12, the marked (highlighted edge) combinations are outside the optimum PPR interval.

\section{Out-of-plane deflection}

In the laboratory test, the surface was deflected in increments of $10 \mathrm{~mm}$ from 0 to $30 \mathrm{~mm}$. The strain was determined in the same sections as in the above analysis where no deflections were applied (vertical sections right in front of the camera).

\section{Direct contact strain measurements}

The readings from the extensometers and strain gauges were compared to check the validity of the two types of strain measurements based on direct contact to the surface. The extensometer readings were in average $19.3 \%$ higher than the values obtained by strain gauges at the same locations. A probable cause of this is that the board surface had to be grinded down (removing the paint layers) to the raw wood in order to attach the strain gauges, and hereby the board thickness was reduced compared to areas with painted surface. This variation shows that even contact measurement methods can provide deviations and underlines the complexity in large surface measurements, even in laboratory conditions. Furthermore, the 
312 results of the strain measured in the middle of the boards and in the side of the boards gave similar results,

313 which indicate that the boards were deflected evenly over the width.

\section{DIC strain measurements}

316 The strains measured with DIC (without the corrections included) were higher than the strain gauge and

317 extensometer measurements around mid-span, and lower closer to the supports. This was expected, due to 318 the shape of the developed total correction curve (from Figure 4). In the top of Figure 13, a digital 319 photograph of the evaluated board is seen with the position of the vertical section for evaluation, and the 320 location of the strain gauges and extensometers. Below the photograph is a full field plot from GOM 321 Correlate of the vertical strains of the entire surface when $30 \mathrm{~mm}$ out-of-plane mid-span deflection was 322 applied. The shown strain plot is based on direct non-corrected measurements from the captured digital 323 photographs. In the bottom of Figure 13, as an example, the strain distribution of the shown vertical section 324 is presented for 10, 20 and $30 \mathrm{~mm}$ mid-span deflection of the board.

325 The tendency to enlarged (pseudo) strain due to out-of-plane deflections in the chosen section was seen 326 both vertically and horizontally. For the horizontal direction, the theoretical strain for the setup was zero 327 (which was also, what the horizontal strain gauges measured). However, due to the deflection towards the 328 camera, a large tensile pseudo strain was present as well. Hypothetically, this strain ought to be similar to 329 the out-of-plane strain correction in equation 2, which was approximately the case in the performed tests.

330 The tests showed also that, areas of the evaluated surface could be difficult for the DIC-software to 331 recognize when the out-of-plane deflection was applied, and erroneous strains could be seen horizontally 332 or vertically in the strain plots. The reason for this type of error was the chosen pattern, which was (in some 333 zones) not "random” enough. Nonetheless, the problem only had minor influence on the evaluations of the 334 strain. 


\section{Correction of out-of-plane deflection}

337 Figure 14 shows the measured vertical strains in the section, for an out-of-plane deflection of $20 \mathrm{~mm}$ at 338 mid-span of the plate. The example is for: Wide-angle lens camera, $3.8 \mathrm{~m}$ camera distance, and $10 \mathrm{~mm}$ 339 circle diameter pattern (the combination is within the optimal PPR-interval). A 120x120 pixels subset size 340 is used. Furthermore, the figure shows the total correction (including both lens distortion, surface rotation 341 and out-of-plane deflection) and the measured strains from the applied foil strain gauges, which were 342 positioned next to the analyzed DIC-section. The strain gauge readings are presented on the secondary axis. 343 Theoretically, the relation should be:

$$
\varepsilon_{\text {true }}=\varepsilon_{\text {strain gauge }}=\varepsilon_{\text {DIC }}-\varepsilon_{\text {rot }}-\varepsilon_{\text {oop }}
$$

In the example in Figure 14 at mid-span, the true strain can be calculated to be $0.54885 \%-0.53276 \%$ $=0.0161 \%$, where the strain gauge in the same position measured a strain of $0.0174 \%-$ a deviation of 7 $\%$ from the strain gauge reading. The standard deviation of the non-corrected DIC-strain was $0.0015 \%$.

For all combinations of parameters, the precision at mid-span was good, but the tests also showed that the precision between mid-span and support deviated more. Since the level of the total correction could be more than a factor 30 larger than the true strain, the true strain was sensitive to the precision of the correction, and hence, highly sensitive to the precision of the deflection measurements by the LVDT's.

Positioning of the LVDT's had to be very precise, and more measuring points could therefore be beneficial. This seems to be the main reasons why it was difficult to achieve a perfect fit between the positioned below the directly measured DIC strains in most areas, as expected.

In Figure 14, parts of the correction curve is positioned above the directly measured DIC-strains, which 
was not perfectly symmetrical, which was the basis of the calculation of the correction curve. The strain gauge measurements were non-symmetric as well, which also indicate that the board did not deflect as 362 would be expected theoretically.

363 Consequently the laboratory testing, highlights some of the governing parameters which affects the 364 sensitivity of the method related to strain measurements of 2D DIC corrected for out-of-plane deflection 365 and surface rotation.

\section{IN-SITU TESTS}

In-situ tests during concrete bridge load testing were performed in two occasions in Denmark in late summer 2016 and winter 2017. At both tests, the bridge was safely loaded via a loading rig in a number of pre-defined tempi (semi deformation controlled loading), and the corresponding deflections were measured

371 as well, Schmidt et al. (2018). The load application setup is seen in Figure 15.

372 In both tests, 2D DIC was applied to the underneath bridge surface, as well as other monitoring 373 equipment. Furthermore, the bridge from 2017 was loaded in three sub-tests: One test, where the full bridge 374 width was loaded, and two tests of cut-out longitudinal strips of the bridge deck.

\section{Bridge specifications}

The tested bridges were identical one span bridges ( $9 \mathrm{~m}$ and $11 \mathrm{~m}$ span) and consisted of a number of (theoretically) simply supported pre-fabricated, pre-tensioned beams. The beams were overturned T-cross sections, and in-situ concrete was cast on top of the beams. A bitumen membrane was applied above the in-situ concrete, and finally, a layer of protecting concrete was applied before the asphalt layers. The buildup is depicted in Figure 16.

382 The bottom slab surfaces were smooth and the raw concrete had adequate contrast for DIC-

383 measurements. Below both bridges were rural roads, where the tri-pods with cameras were positioned. The 384 underpasses were closed during the load testing. 


\section{Test method and parameters}

Each test was conducted by first positioning monitoring equipment for deflection- (land surveyor, LVDT's and distance lasers) and strain measurements (strain gauges) under the bridge. The type of equipment was the same as for the laboratory tests, and the distance lasers were of type Leutze ODSL 30. 17 for a photograph of the setup for the 2017 winter test of the full width bridge.

The distance from the wide-angle lens camera to the surface was approximately $3.8 \mathrm{~m}$ in both tests. The

$\mathrm{m}$ from the surface. The position and ranges of the DIC-cameras, and the number of applied deflection and in the summer of 2016 had a setup similar to the full-width bridge test in the figure, except for the span being $11 \mathrm{~m}$ and the wide-angle lens camera being rotated 90 degrees.

\section{EVALUATION OF PRECISION OF IN-SITU TESTS}

The in-situ test was evaluated using the same approach as the laboratory tests, by DIC-analysis of a section along the span. A full longitudinal section was assessed before any deflection was applied to the surface. The Summer test 2016 was used as an example, and in Figure 19, it is seen, how the precision is best, closest to where the camera was perpendicular to the surface. This is of significant importance when 
Results from all available in-situ tests over an entire longitudinal section length are shown in Figure 20.

411 The evaluation was performed for subset sizes of 40x40 pixels, 80x80 pixels and 120x120 pixels, and the

412 joining lines are applied to achieve an overview. The strain standard deviation of the Summer test 2016

413 example, was approximately $0.05 \%$ for subset size 120 pixels. Note that all the precisions by the wide-

414 angle lens camera were based on photographs captured from the same distance, and of the same type of 415 concrete surface. Hence, the discrepancy of the strain standard deviations, seen in Figure 20, was due to the 416 specific local weather and light conditions at the time of the tests.

417 The sections are chosen to be directly above the cameras (see Figure 18). In the evaluation, there has 418 been accounted for areas of the surfaces, where e.g. cables for the LVDT's were crossing the evaluated part 419 of the photograph. This could potentially have affected the strain readings (as described earlier).

\section{In-situ corrections of out-of-plane deflection with surface rotation}

In regard to corrections for out-of-plane deflection and surface rotation in the in-situ tests, the example from the Summer 2016 test is depicted in Figure 21 during load testing (in the figure, the load was 2444

$424 \mathrm{kN}$ and the mid-span deflection was $6.6 \mathrm{~mm}$ ). The points of the DIC-results are scattered around the trend 425 line. These deviations may be considered as the discrepancies in PPR in different location over the evaluated 426 section length, where some areas have a more optimal PPR than others, in regard to the precision. 427 Consequently, if the PPR can be optimized in these positions, it is deemed that they will move closer to the 428 trend line. The best trend line, in the specific case, is a second degree polynomial. The two curves seem to 429 have a correct relation, since the true strain is calculated as the difference between the measured strain and 430 the corrected strain along the span, cf. Equation 7. laboratory tests showed significantly lower standard deviations, when compared to the field test results. 
The discrepancy does, however, not mean that the DIC-equipment has an inadequately low strain precision for field use, but rather that the method has a very high level of strain precision under controlled laboratory circumstances. order of magnitude $0.05 \%$, while the interval of the standard deviations for the laboratory tests at the same subset size was from $0.0015 \%$ to $0.0072 \%$, depending on the combination of parameters.

The regular lens camera showed higher standard deviations in the laboratory tests compared to the wideangle lens camera (in the interval from $0.005 \%$ to $0.013 \%$ ), and this indicated that the image quality of the full field Canon 6D had an influence as well, since the focal lengths used were almost the same for both cameras (16mm and 18mm, respectively). Even though the regular lens camera showed less good strain precision in the DIC laboratory tests, the precision was similar to the wide-angle lens camera in the in-situ for a subset size of 40 pixels, for the wide-angle lens camera. Two specific parameter combinations gave a similar standard deviation, when comparing the laboratory- and in-situ tests, which is worth noticing: tests, where the regular lens camera was positioned closer to the surface than the wide angle-lens camera.

$$
\text { A } 0.05 \% \text { standard deviation, similar to the in-situ precision, was found in the laboratory tests, but only }
$$
1) The combination of subset size 40, $2.6 \mathrm{~m}$ camera distance, and $10 \mathrm{~mm}$ circle pattern gave a strain standard deviation of $0.047 \%$. That specific combination of parameters gave a PPR of 14 pixels per circle diameter.

2) The combination of subset size 40, $3.8 \mathrm{~m}$ camera distance, and $3 \mathrm{~mm}$ circle pattern gives a strain standard deviation of $0.046 \%$. A PPR for that combination was 3 pixels per circle diameter.

Both combination were lying outside the boundaries of the optimal PPR interval found in the laboratory tests, see the example in Figure 12.

In Figure 22, an example is given to clarify the difference between the grey distribution of typical appearing subsets from the in-situ tests and the laboratory tests. The example is based on photographs by the wide-angle lens camera at a distance of $3.8 \mathrm{~m}$, and the laboratory subset are with $10 \mathrm{~mm}$ pattern circle 
diameter. Given the random choice of location, the standard deviations in this example are coincidentally higher than the average standard deviations. In the figure, the texture of the raw concrete surface is clearly finer than the comparable pattern of the painted boards in the laboratory. This indicates that the raw concrete surface is most comparable to a painted pattern with a PPR below the optimal interval. An optimization of the field precision could therefore be expected by raising the PPR by either testing with a smaller camera distance (which can be an in-situ challenge) or by having a higher camera resolution, in combination with 467 a further increase in the subset size. It should be noted that the optimal strain precision, is not at the same 468 time equal to the earliest detection of cracks, which would require another type of study. Consequently, such investigation is ongoing and not a part of this paper.

\section{CONCLUSION}

472 The ongoing Danish bridge load testing program involves the use of 2D Digital Image Correlation monitoring equipment applied to the underneath surface of concrete bridges during load testing. The presented purpose of the researched 2D DIC system is to evaluate some of the governing parameters affecting the strain precision and additionally provide some user boundaries. The method is deemed an important tool to provide one or more stop criteria, when used in relation to in-situ proof loading of concrete bridges. In-situ testing and related DIC monitoring is extremely challenging compared to laboratory testing, due to environmental conditions, short testing time, structural size, light conditions, accessibility etc. The paper proposes a method, which can be used as an input, regarding strain precision and out-of-plane pseudo strain corrections, before addressing DIC-monitoring in conjunction with in-situ bridge load testing.

It is seen in this study that the bridge surface deflects towards the camera as load is applied on the top surface of the bridge deck. A wide-angle lens DSLR-camera was applied, to achieve the largest possible ROI. The wide-angle lens camera was compared to another DSLR-camera with a regular lens, and the strain precision of both was analyzed in laboratory tests and compared to examples from field tests. 
In the laboratory tests, where wooden boards were painted (in representative grey concrete nuances) with circle patterns, the following parameters were studied: Camera to surface distance, subset size, pattern circle diameter, and camera type. The laboratory tests showed that the Pattern Pixel Relation (PPR), which is the number of pixels per circle diameter, was an important indicator of the DIC precision. The lowest level of standard deviation in the evaluated sections on the boards, seemed to be within an interval from 4 to 9 pixels per circle diameter, regardless of the camera type.

The wide-angle lens camera was studied in regard to the strain section precision over the width of the photographs, and the precision was best, where the camera direction was perpendicular to the surface (right in front of the camera) and decreased towards the sides of the photographs. This was due to the higher level of lens distortion correction in the sides.

The DIC-evaluation of an example field tested bridge showed a higher standard deviation of the strain in sections on the raw concrete surfaces, compared to most of the laboratory test results. Nevertheless, the level of precision by wide-angle lens camera, from the in-situ bridges were comparable to laboratory tests pixels per circle diameter, which is smaller and larger than the boundaries of optimal proposed PPR interval. When analyzing the raw concrete surfaces against the laboratory pattern at close proximity at subset level, it was clear that the raw concrete had a much finer texture, indicating a texture corresponding to a low PPR. for the field tests could be done by e.g. moving the camera closer to the surface (which would reduce the ROI), or purchasing a camera housing with an even better resolution.

In addition to the investigations of the non-deflected surfaces, the out-of-plane correction, including surface rotations, were applied in both the laboratory tests, and in the in-situ bridge tests. In both cases, the 508 correction curves fitted well with the directly measured DIC strains, which indicate that the method seems 509 correct. It was shown that the proposed new contribution to the strain correction from the surface rotation 510 was of significant importance, and must be included in 2D DIC evaluations of larger surfaces with out-of511 plane bending. Furthermore, it was noticed that the precision of the out-of-plane deflection and surface 
rotation corrections were extremely sensitive to correctly measured deflections (for instance by LVDT’s),

513 and placing of the equipment. The strain analysis differs from a crack initiation analysis, which is therefore

514 not a part of this paper.

515 The findings have provided important information in regard to understanding monitoring thresholds as

516 well as means to optimize the strain precision further. Ongoing research therefore concerns optimization in 517 regards to multidirectional strain evaluation combined with crack initiation detection.

\section{ACKNOWLEDGEMENT}

520 The authors would like to acknowledge the great cooperation of the technical staff at the testing facilities

521 at DTU. Furthermore, we would like to thank our project partners at COWI and at The Danish Road 522 Directorate for the funding and the excellent team work in the "Bridge Load Testing Project".

\section{REFERENCES}

525 Bornert, M. et al. (2009). “Assessment of Digital Image Correlation Measurement Errors: Methodology and Results.” Experimental Mechanics, 49(3), 353-70.

Bruck, H. A., McNeill, S. R., Sutton, M. A., and Peters, W. H. (1989). "Digital Image Correlation Using Newton-Raphson Method of Partial Differential Correction." Experimental Mechanics, 29(3), 261-

Chen, Z., Quan, C., Zhu, F., and He, X. (2015). “A Method to Transfer Speckle Patterns for Digital Image Correlation.” Measurement Science and Technology, 26(9), 95201.

532 Crammond, G., Boyd, S. W., and Dulieu-Barton, J. M. (2013). “Speckle Pattern Quality Assessment for Digital Image Correlation.” Optics and Lasers in Engineering, 51(12), 1368-78.

534 GOM, (2018). GOM Correlate Professional.

535 Halding, P. S., Hertz, K. D., Schmidt, J.W., and Kennedy, B.J. (2016). "Full-scale load tests of Pearl$536 \quad$ Chain arches." Eng Structures, 131, 101-114. 
Halding, P. S., Schmidt, J. W., and Christensen, C. O. (2018). "DIC-monitoring of full-scale concrete bridge using high-resolution wide-angle lens camera." Proc., IABMAS Melbourne 2018, Powers, Frangopol, Al-Mahaidi and Caprani, ed., Taylor \& Francis Group, London, 1492-99.

Halding, P. S., Schmidt, J. W., Jensen, T. W., and Henriksen, A. (2017). "Structural response of full-scale concrete bridges subjected to high load magnitudes." Proc., $4^{\text {th }}$ Conference in Smart Monitoring, Assessment and Rehabilitation of Civil Structures, 266.

Hoult, N. A., Take, W. A., Lee, C., and Dutton, M. (2013). "Experimental Accuracy of Two Dimensional Strain Measurements Using Digital Image Correlation.” Eng Structures, 46, 718-26.

Lecompte, D., Bossuyt, S., Cooreman, S., Sol, H., and Vantomme, J. (2007). “Study and Generation of Optimal Speckle Patterns for DIC.” Proc., of the SEM Annual Conference and Exposition on Experimental and Applied Mechanics, Society for Experimental Mechanics, 3, 1643-49.

Lecompte, D. et al. (2006). “Quality Assessment of Speckle Patterns for Digital Image Correlation.” Optics and Lasers in Engineering, 44(11), 1132-45.

Pan, B., Yu, L., and Wu, D. (2014). “High-Accuracy 2D Digital Image Correlation Measurements Using Low-Cost Imaging Lenses: Implementation of a Generalized Compensation Method.” Measurement Science and Technology, 25(2), 25001.

Park, J., Yoon, S., Kwon, T. H., and Park, K. (2017). “Assessment of Speckle-Pattern Quality in Digital Image Correlation Based on Gray Intensity and Speckle Morphology.” Optics and Lasers in Engineering, 91, 62-72.

Photoshop (2018), Adobe Photoshop CS6.

Ruocci, G. et al. (2016). "Digital Image Correlation and Noise-filtering Approach for the Cracking Assessment of Massive Reinforced Concrete Structures." Strain, 52(6), 503-521.

Schmidt, J. W., Halding, P. S., Jensen, T. W., and Engelund, S. (2018). "High Magnitude Loading of Concrete Bridges." Evaluation of Concrete Bridge Behavior through Load Testing - International Perspectives - 2018, ACI, SP 323-9.

Schmidt, J. W., Hansen, S. G., Barbosa, R. A., and Henriksen, A. (2014). "Novel shear capacity testing of 
ASR damaged full scale concrete bridge." Eng Structures, 79, 365-374.

564 Schreier, H., Orteu J. J., and Sutton M. A. (2009). "Image Correlation for Shape, Motion and Deformation 565 Measurements: Basic Concepts, Theory and Applications." Springer US, 1-321.

566 Sutton, M. A. et al. (2017). “Recent Progress in Digital Image Correlation: Background and 567 Developments since the 2013 W M Murray Lecture.” Experimental Mechanics, 57(1), 1-30.

568 Sutton, M.A., Wolters, W. J., Peters, W. H., Ranson, W. F., and McNeill, S. R. (1983). “Determination of 569 570 Strain States.” Applied Mechanics and Materials, 70, 177-82.

575 Waterfall, P., McCormick, N., and Owens, A. (2014). “Optical Imaging for Low-Cost Structural 576 Measurements.” Proc., The Institution of Civil Engineers: Bridge Engineering, Thomas Telford Services Ltd, 167, 33-42. image correlation by measuring rigid body displacement." Optical Engineering, 45(2), 023602. Using Digital Image Correlation.” Experimental Techniques, 31(1), 34-40. 